\title{
Determination of the LO phonon energy by using electronic and optical methods in AlGaN/GaN
}

\author{
Ozlem Celik $^{1 *}$, Engin Tiras ${ }^{1}$, Sukru Ardali ${ }^{1}$, Sefer B. Lisesivdin ${ }^{2}$, Ekmel Ozbay $^{3}$ \\ 1 Department of Physics, Faculty of Science, Anadolu University, \\ Yunus Emre Campus, 26470 Eskisehir, Turkey \\ 2 Department of Physics, Faculty of Science and Arts, Gazi University, \\ Teknikokullar, 06500 Ankara, Turkey \\ 3 Nanotechnology Research Center, Department of Physics, and Department of Electrical and Electronics Engineering, Bilkent \\ University, \\ Ankara, Turkey
}

Received 20 May 2011; accepted 20 October 2011

\begin{abstract}
:
The longitudinal optical (LO) phonon energy in $A I G a N / G a N$ heterostructures is determined from temperature-dependent Hall effect measurements and also from Infrared (IR) spectroscopy and Raman spectroscopy. The Hall effect measurements on AIGaN/GaN heterostructures grown by MOCVD have been carried out as a function of temperature in the range $1.8-275 \mathrm{~K}$ at a fixed magnetic field. The IR and Raman spectroscopy measurements have been carried out at room temperature. The experimental data for the temperature dependence of the Hall mobility were compared with the calculated electron mobility. In the calculations of electron mobility, polar optical phonon scattering, ionized impurity scattering, background impurity scattering, interface roughness, piezoelectric scattering, acoustic phonon scattering and dislocation scattering were taken into account at all temperatures. The result is that at low temperatures interface roughness scattering is the dominant scattering mechanism and at high temperatures polar optical phonon scattering is dominant.
\end{abstract}

PACS (2008): 72.20.Fr; 73.50.Dn; 72.20.Dp; 78.30.Fs

Keywords: $\quad$ Hall effect $\bullet \mathrm{LO}$ phonon energy $\cdot \mathrm{AlGaN} / \mathrm{GaN} \cdot$ Raman spectra $\bullet$ infrared spectra

(C) Versita Sp. z o.o.

\section{Introduction}

Group III-nitride materials are very suitable for applications in high power, high frequency and high temperature electronics $[1,2]$. Due to the large bandgap and thermal

*E-mail: ozlem.c@anadolu.edu.tr properties of GaN it is very useful to operate AlGaN/GaN high electron mobility transistors (HEMTs) [3-6]. In AlGaN/GaN heterostructures, two-dimensional electron gas (2DEG) can be observed at the interface with high sheet carrier density values [7]. The mobility and density of 2D electrons are very important transport parameters for device performance [8]. The mobility of electrons in these structures is limited by a combination of scattering mechanisms [9]. In AlGaN/GaN HEMTs, the electron mobility 
is limited by polar optical phonons at room temperature [10]. However, different scattering mechanisms, including interface roughness scattering, studied by other research groups are effective at low temperatures $[8,11]$. The investigation of the polar optical phonon energies and electronphonon scattering rates in AlGaN/GaN heterostructures is important in order to understand how these devices operate at high electric fields where the electron scattering with longitudinal optical (LO) phonons dominates the conductivity.

In this paper we have determined the LO phonon energy of GaN using two techniques: one optical and the other electronic. Raman and Infrared spectroscopy measurements are the optical techniques and temperaturedependent Hall effect measurements is the electronic technique. Optical techniques give the value of LO phonon energy directly from the spectra. To obtain the LO phonon energy from the temperature-dependent Hall effect measurements, appropriate theoretical expressions for the energy and momentum relaxation rates have to be used. In this study the LO phonon energy in GaN is determined from IR, Raman and Hall effect measurements on the same AlGaN/GaN heterostructure sample.

\section{Experimental}

The AlGaN/GaN heterostructure was grown by the metal organic chemical vapor deposition (MOCVD) technique on a sapphire substrate. The layers consisted of a $320 \mathrm{~nm}$ AlN buffer layer, followed by a $1.7 \mu \mathrm{m}$ undoped GaN layer, a $1 \mathrm{~nm}$ AlN spacer layer and a $20 \mathrm{~nm} \mathrm{Al}_{x} \mathrm{Ga}_{1-x} \mathrm{~N}(x=0.25)$ layer capped with a $3 \mathrm{~nm} \mathrm{GaN}$. The $\mathrm{Al}_{x} \mathrm{Ga}_{1-x} \mathrm{~N}$ layer was doped with $\mathrm{Si}$, doping density $1018 \mathrm{~cm}^{-3}$. The 2DEG was formed at the interface between the undoped GaN layer and AIN spacer. The sample was grown in the wurtzite structure. The layer structure of the sample used in this study is shown in Table 1. During the growth, the sample parameters including doping density, alloy fractions and layer thicknesses were estimated from the calibrated charts for the specific growth conditions and materials. After the growth, these parameters were measured for each wafer, using standard characterization techniques such as photoluminescence, scanning transmission electron spectroscopy, capacitance-voltage profiling and energy dispersive $\mathrm{x}$-ray analysis $[12,13]$.

The IR spectra were obtained at room temperature by using a Bruker Optics IFS66v/S FT-IR system in the range $4000-40 \mathrm{~cm}^{-1}$. The Raman spectra were obtained at room temperature by using a Bruker Optics FT-Raman Scope III system. As an excitation source, a $532 \mathrm{~nm}$ wavelength laser was applied in the sample growth
Table 1. Layer structure of the $\mathrm{Al}_{x} \mathrm{Ga}_{1-x} \mathrm{~N} / \mathrm{GaN}$ heterostructure sample.

\begin{tabular}{cc}
\hline Layer & Thickness $(\mathrm{nm})$ \\
\hline $\mathrm{Al}_{x} \mathrm{Ga}_{1-x} \mathrm{~N}(x=0.25$, doped barrier) & 3 \\
$\mathrm{AIN}$ (spacer) & 20 \\
GaN (undoped) & 1700 \\
AIN (buffer) & 320 \\
Sapphire (substrate) & \\
\hline
\end{tabular}

direction (c-axis).

A square-shaped sample $(5 \times 5 \mathrm{~mm})$ with Van der Pauw geometry was used for Hall effect and magnetoresistance measurements. These measurements were performed in a cryogen-free superconducting magnet system (Croyogenics Ltd.) using a conventional DC technique in combination with a constant current-voltage source Keithley 2400, switch system Keithley 7100, nanovoltmeter Keithley $182 \mathrm{~A}$ and temperature controller Lakeshore 340. The current flow was in a plane that is perpendicular to the sample growth direction. A static magnetic field $(B=1$ T) was applied to the sample perpendicular to the current plane. The longitudinal resistance $\left(R_{x x}\right)$ along the applied current and the Hall resistance $\left(R_{x y}\right)$ were measured as a function of temperature from 1.89 to $275 \mathrm{~K}$. The voltage applied to the sample was kept low enough to ensure ohmic conditions, in order to avoid carrier heating. All of the measurements were carried out in darkness. The Hall mobility $\left(\mu_{H}\right)$ and the sheet carrier density (NS) were obtained using following equations

$$
\begin{gathered}
R_{x y}=\frac{B}{N_{s} e} \\
\mu_{H}=\frac{1}{N_{s} R_{x x}}
\end{gathered}
$$

\section{Scattering mechanisms}

The scattering mechanisms of two-dimensional (2D) carriers in III-V heterostructures are well described [8, 10, 11, 14-19]. The scattering mechanisms we used in $\mathrm{Al}_{0.25} \mathrm{Ga}_{0.75} \mathrm{~N} / \mathrm{GaN}$ heterostructures are: polar optical phonon scattering, acoustic phonon scattering due to deformation potential coupling, acoustic phonon scattering due to piezoelectric coupling, background impurity scattering, dislocation scattering, ionized impurity scattering, and interface roughness scattering. The total electron mobility $\left(\mu_{t o t}\right)$ can be calculated from the scattering-limiting 
mobilities $\left(\mu_{j}\right)$ by using Matthiessen's rule:

$$
\frac{1}{\mu_{t o t}}=\sum \frac{1}{\mu_{j}}
$$

with

$$
\frac{1}{\mu_{j}}=\frac{e \tau_{j}}{m^{*}}
$$

where e is the electronic charge, $\tau_{j}$ is the momentum relaxation time defined for each scattering process and $m^{*}$ is the electron effective mass.

The analytical expressions for the scattering mechanisms mentioned above are summarized below.

\subsection{Polar optical phonon scattering}

At high temperatures polar optical phonon scattering is dominant in GaN, a highly polar material [19], and due to the large optical-phonon energy, scattering of electrons by optical phonons is inelastic $[10,16]$. It has been shown that the three-dimensional (3D) approach to polar opticalphonon scattering is justifiable for the 2DEG (Refs [20 23]). The mobility limited by the polar optical phonon scattering (in SI units) can be given by [21]:

$$
\mu_{P O}=\frac{4 \pi \varepsilon_{0} \hbar}{3 e N m^{*}}\left(\frac{2 \hbar}{m^{*} \omega_{L O}\left(1+\hbar \omega_{L O}\right) / E_{g}}\right)^{\frac{1}{2}} \frac{I_{2}\left(\frac{k_{B} T}{E_{g}}\right)}{l_{1}\left(\frac{k_{B} T}{E_{g}}\right)}
$$

where

$$
\frac{1}{\varepsilon^{*}}=\frac{1}{\varepsilon_{\infty}}-\frac{1}{\varepsilon_{s}}
$$

with $\hbar(=h / 2 \pi)$ is the Planck constant, $\varepsilon_{\infty}$ is the high frequency dielectric constant, $\varepsilon_{s}$ is the static dielectric constant, $\varepsilon_{0}$ is the permittivity of vacuum, $N=$ $1 /\left(e^{\hbar \omega_{L O} / k_{B} T}-1\right)$ is the Planck distribution function, $\omega_{L O}$ $s$ the optical phonon frequency, $E_{g}$ is the band gap energy of GaN, $T$ is the absolute temperature, $k_{B}$ is the Boltzmann constant, and

$$
\begin{aligned}
& I_{1}(\gamma)=\int_{0}^{\infty}(1+2 \gamma x) \sqrt{x(1+\gamma x)} \exp (-x) d x \\
& I_{2}(\gamma)=\int_{0}^{\infty}[x(1+\gamma x)]^{\frac{3}{2}}(1+2 \gamma x)^{-1} \exp (-x) d x,
\end{aligned}
$$

where

$$
\gamma=\frac{k_{B} T}{E_{g}} .
$$

\subsection{Acoustic phonon scattering}

The acoustic phonon scattering includes deformation potential scattering and piezoelectric scattering. The mobility expression of deformation potential scattering is [16]:

$$
\mu_{D P}=\frac{e \hbar^{3} \rho b^{\prime} u_{1}^{2}}{m^{* 2} E_{A}^{2} k_{B} T} \frac{1}{I_{A}\left(\gamma_{l}\right)},
$$

where $\rho$ is the mass density of $\mathrm{GaN}, b^{\prime}$ is the effective thickness of the 2D layer in the heterojunction, $u_{l}$ is the velocity of longitudinal acoustic phonons, $E_{A}$ is the acoustic deformation potential, and

$$
I_{A}\left(\gamma_{l}\right)=\left[\left(\frac{4 \gamma_{l}}{3 \pi}\right)^{2}+1\right]^{\frac{1}{2}}
$$

with

$$
v_{l}=\frac{2 \hbar u_{1} k_{F}}{k_{B} T}
$$

where $k_{F}=\sqrt{2 \pi N_{2 D}}$ is the Fermi wavelength of $2 \mathrm{D}$ electrons in the first subband. In highly polar materials such as GaN, the mobility limited by acoustic piezoelectric scattering can be calculated by the relaxation time approach. The ratio of the momentum relaxation time $\left(\tau_{D P}\right)$ for acoustic deformation potential scattering to that $\left(\tau_{P E}\right)$ for acoustic piezoelectric scattering in a $2 \mathrm{D}$ electron gas is given by [16]:

$$
\frac{\tau_{D P}}{\tau_{P E}}=\frac{b^{\prime}}{\pi k_{F}}\left[\frac{9}{32}+\frac{13}{32}\left(\frac{u_{l}}{u_{t}}\right)^{2} \frac{I_{A}\left(\gamma_{t}\right)}{I_{A}\left(\gamma_{l}\right)}\right] \frac{e h_{14}^{2}}{E_{A}}
$$

where $h_{14}$ is the piezoelectric constant, $u_{t}$ is the velocity of transverse acoustic phonons, and

$$
I_{A}\left(\gamma_{t}\right)=\left[\left(\frac{4 \gamma_{t}}{3 \pi}\right)^{2}+1\right]^{\frac{1}{2}}
$$

with

$$
\nu_{t}=\frac{2 \hbar u_{t} k_{F}}{k_{B} T}
$$

The mobility $\left(\mu_{P E}\right)$ limited by piezoelectric scattering can be obtained from [16]

$$
\mu_{P E}=\mu_{D P} \frac{\tau_{P E}}{\tau_{D P}}
$$




\subsection{Background impurity scattering}

The mobility limited by background impurity scattering can be obtained from [24]

$$
\mu_{B I}=\frac{8 \pi \hbar^{3} \varepsilon_{S}^{2} k_{F}^{2} I_{B}(\beta)}{e^{3} m^{*} 2 N_{B I}} .
$$

Here $N_{B}$ is the $2 \mathrm{D}$ impurity density in the potential well due to background impurities and/or interface charge, and

$$
I_{B}(\beta)=\int_{0}^{\pi} \frac{\sin ^{2} \theta d \theta}{(\sin \theta+\beta)}
$$

where $\theta$ is the scattering angle, and

$$
\beta=\frac{2 e^{2} m^{*}}{8 k_{F} \pi \varepsilon_{S} \hbar^{2}}
$$

\subsection{Dislocation scattering}

The expression for the dislocation scattering for a degenerate 2DEG can be obtained from [25]

$$
\mu_{\text {dis }}=\frac{16 \pi k_{F}^{4} \hbar^{3} \varepsilon_{S}^{2} c^{a s t 2}}{N_{\text {dis }} m^{* 2} e^{3} l_{t}}
$$

where $N_{d i s}$ is the charge dislocation density, $c^{*}(=5.186$ $\AA$ ) is the lattice constant in the (0001) direction of wurtzite GaN, and

$$
I_{t}=\frac{1}{2} \xi^{2} \int_{0}^{1} \frac{d u}{\left(1+\xi^{2} u^{2}\right) \sqrt{1-u^{2}}}
$$

with $\xi=2 k_{F} / q_{T F}, q_{T F}=2 / a_{B}$ is the $2 \mathrm{D}$ Thomas Fermi wave vector, and $a_{B}$ is the effective Bohr radius.

\subsection{Ionized impurity scattering}

The expression for the mobility due to ionized impurity scattering [26]

$$
\mu_{l}=\frac{24 \pi^{3} \varepsilon_{S}^{2} \hbar^{3} N_{3 D}}{e^{3} m^{* 2} N_{i o n}[\ln (1+y)-y /(1+y)]},
$$

where

$$
y=\frac{2 \pi^{\frac{8}{3}} 3^{\frac{1}{3}} \hbar^{2} \varepsilon_{S}\left(N_{3 D}\right)^{\frac{1}{3}}}{e^{2} m^{*}}
$$

Here $N_{i o n}$ is the density of ionized impurities which is in the order of $1014 \mathrm{~cm}^{\text {âAş3 }}, N_{3 D}\left(=N_{2 D} / L_{z}\right)$ is the 3D electron concentrations, and $L_{z}$ is the quantum well width.

\subsection{Interface roughness scattering}

Interface roughness (IFR) in III-V heterostructures has been described by a Gaussian distribution of lateral size $(\Lambda)$ and width $(\Delta)$ of the IFR. The electron mobility limited by IFR scattering can be calculated using [16]

$$
\mu_{I F R}=\frac{e}{m *}\left[\left(\frac{e^{2} N_{2 D} \Lambda}{2 \varepsilon_{S}}\right)^{2} \frac{m *}{\hbar^{3}} J(k)\right]^{-1}
$$

Here

$$
\left.J(k)=\int_{0}^{1} 2 k\right) \frac{\exp \left(-q^{2} \wedge^{2} / 4\right)}{2 k^{3}(q+q s)^{2} \sqrt{1-(q / 2 k)^{2}}} q^{4} d q
$$

where $q=2 k \sin (\theta / 2), k$ is the electron wave vector. and

$$
q_{s}=\frac{e^{2} m^{*}}{2 \pi \varepsilon_{s} \hbar^{2}} F(q)
$$

is the screening constant in which $F(q)$ is the form factor defined by

$$
F(q)=\int_{0}^{\infty} \int_{0}^{\infty}\left[\psi(z) \psi\left(z^{\prime}\right)\right]^{2} e^{-q\left|z-z^{\prime}\right| d z^{\prime} d z}
$$

where $\psi(z)$ is the Fang-Howard variational wave function [27].

\section{Results and discussion}

According to Raman spectroscopy of a crystal with wurtzite structure the left $A_{1}, E_{1}$, two $B_{1}$ and two $B_{2}$ modes are optical modes of vibration. The $A_{1}$ and $E_{1}$ modes correspond to polar optical vibrations and net electric dipole is formed in each unit cell for polar optical vibrations. However, the $B_{1}$ and $E_{2}$ modes are non-polar optical vibrations. The polar modes are active for IR spectroscopy. Two $E_{2}$ modes and similarly the $B_{1}$ modes are labeled as low and high modes, because for the low mode the displacement of the atoms is shear and for the high mode the displacement of the atoms is compression [28]. The LO phonon energy in GaN can be determined from the wave number of the $A_{1}$ mode from Raman and IR spectra.

Figure 1 shows the room temperature Raman (lower) and infrared spectra (upper) for $\mathrm{Al}_{0.25} \mathrm{Ga}_{0.75} \mathrm{~N} / \mathrm{GaN}$ heterostructures recorded in the grown axis backscattering configurations. The $E_{2}$ and $A_{1}(L O)$ modes for $\mathrm{GaN}$ are shown in this configuration. There is a sharp and strong 


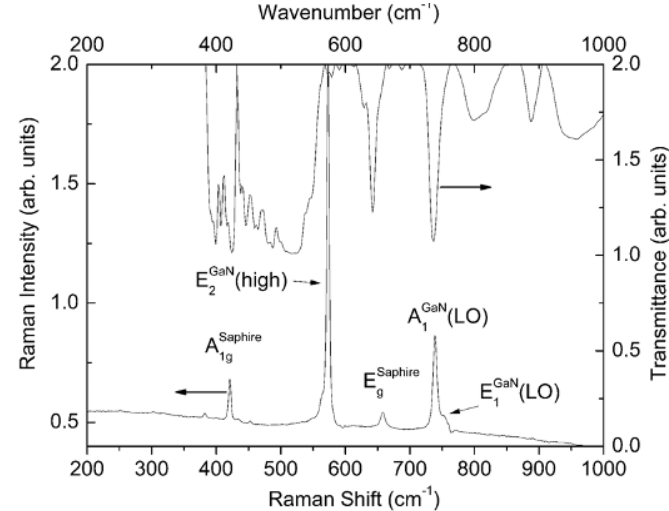

Figure 1. IR (upper) and Raman (lower) spectra for an $\mathrm{Al}_{0.25} \mathrm{Ga}_{0.75} \mathrm{~N} / \mathrm{GaN}$ heterostructure measured at room temperature.

peak at $571.5 \mathrm{~cm}^{-1}$, known as the non-polar high frequency $E_{2}$ mode, which implies strong correlation between the $\mathrm{Ga}$ and $\mathrm{N}$ atoms on the c-plane [29, 30]. The polar vibrations $A_{1}$ and $E_{1}$ observed at 736 and $748.5 \mathrm{~cm}^{-1}$ respectively, also correspond to correlation between Ga and $\mathrm{N}$ atoms. Since the penetration depth of the source light (wavelength $532 \mathrm{~nm}$ ) is longer than the thickness of the coated wafer on the sapphire substrate, the $A_{1 g}$ and $E_{g}$ modes originating from the sapphire substrate are observed at approximately 417.5 and $642.2 \mathrm{~cm}_{-1}$, respectively. The $A_{1}(L O)$ mode is observed at the wave-number $736 \mathrm{~cm}^{-1}$ in both the Raman spectra and IR spectra. The energy of LO phonons in GaN ( $\hbar \omega_{L O}=91.2 \mathrm{meV}$ ) is determined using $\hbar \omega_{L O}=\hbar c \bar{v}$, where $c$ is the speed of light and is the wave number of the $A_{1}(L O)$ mode. This value for $\hbar \omega_{L O}$ is very close to that reported previously $[31,32]$.

Figure 2 shows the temperature dependence of the longitudinal resistance $\left(R_{x x}\right)$ and Hall resistance $\left(R_{x y}\right)$ measured for the $\mathrm{Al}_{0.25} \mathrm{Ga}_{0.75} \mathrm{~N} / \mathrm{GaN}$ heterostructure. The variations of Hall mobility $\left(\mu_{H}\right)$ and sheet carrier density $\left(N_{S}\right)$ with temperature, as calculated from the experimental $R_{x x}(\mathrm{~T})$ and $R_{x y}(\mathrm{~T})$ data using equations (1) and (2), are shown in Figure 3. At low temperatures the sheet carrier density is essentially independent of temperature, however, at high temperatures the sheet carrier density increases with increasing temperature due to thermally generated bulk related carriers. At high temperatures the Hall mobility decreases with increasing temperature and at low temperatures (below about $50 \mathrm{~K}$ ) the Hall mobility is practically independent of temperature. This behavior reflects the $2 \mathrm{D}$ character of the electrons in the $\mathrm{Al}_{0.25} \mathrm{Ga}_{0.75} \mathrm{~N} / \mathrm{GaN}$ heterostructure.

At high temperatures polar optical phonon scattering is

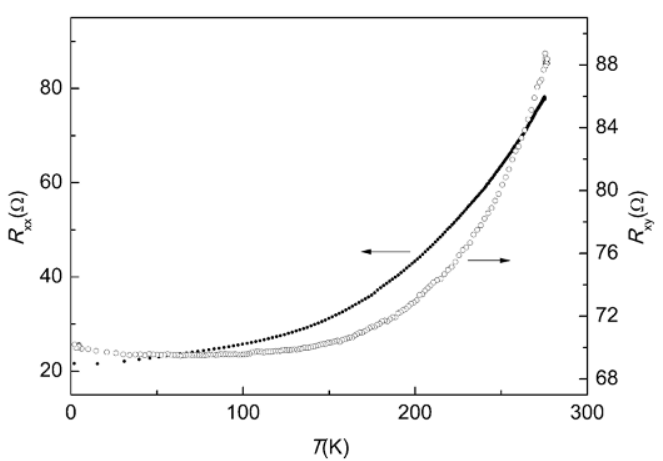

Figure 2. Temperature dependence of the longitudinal resistance $\left(R_{x x}\right)$ and Hall resistance $\left(R_{x y}\right)$ measured at a magnetic field of $1.0 \mathrm{~T}$ for the $\mathrm{Al}_{0.25} \mathrm{Ga}_{0.75} \mathrm{~N} / \mathrm{GaN}$ heterostructure.

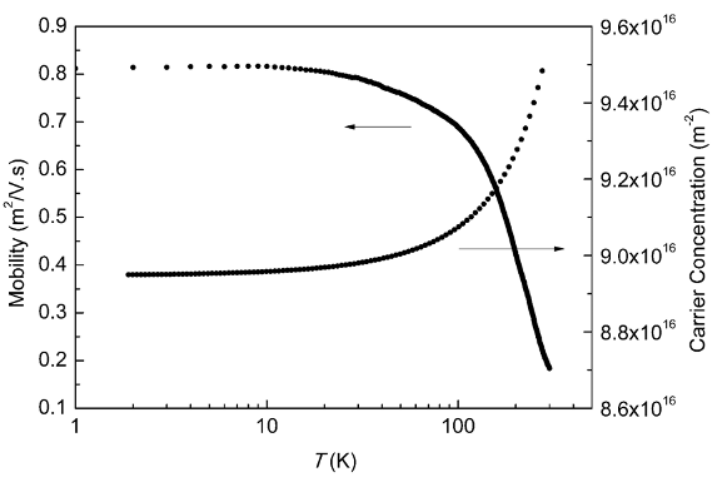

Figure 3. Temperature dependence of the Hall mobility $\left(\mu_{H}\right)$ and the sheet carrier density $\left(N_{S}\right)$ in $\mathrm{Al}_{0.25} \mathrm{Ga}_{0.75} \mathrm{~N} / \mathrm{GaN}$ heterostructure.

the dominant scattering mechanism in GaN [19]. The LO phonon scattering limited mobility $\left(\mu_{L O}\right)$ can be extracted from the measured Hall mobility by rewriting Matthiessen's rule [33] as

$$
\frac{1}{\mu_{L O}}=\frac{1}{\mu_{H}}-\frac{1}{\mu_{0}}
$$

where $\mu_{0}$ is the low-temperature Hall mobility which is independent of temperature and $\mu_{H}$ is the temperaturedependent Hall mobility measured at temperatures above about $150 \mathrm{~K}$. Figure 4 presents a plot of the natural logarithm of $\left(1 / \mu_{H}-1 / \mu_{0}\right)$ versus $1 / T$. The LO phonon energy is determined from the gradient of the straight line, which is the best fit to the experimental data above about 170 $K$. The value $\hbar \omega_{L O}=89 \mathrm{meV}$ determined by this method is in good agreement with that reported previously, 91.2 


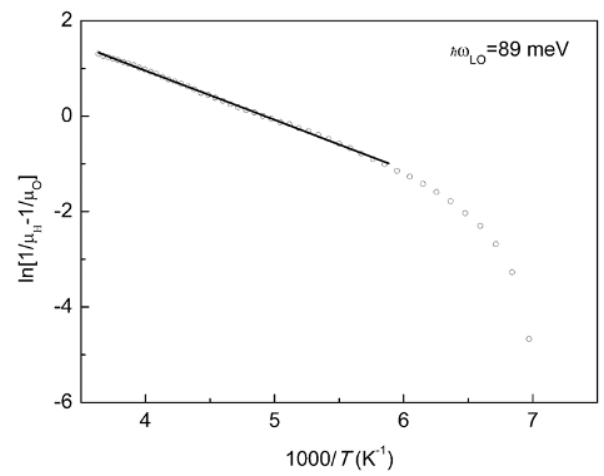

Figure 4. Plot of $\ln \left(1 / \mu_{H}-1 / \mu_{0}\right)$ versus $1000 / T$ for the $\mathrm{Al}_{0.25} \mathrm{Ga}_{0.75} \mathrm{~N} / \mathrm{GaN}$ heterostructure. The LO phonon energy $\left(\hbar \omega_{\llcorner O}=89 \mathrm{meV}\right)$ is obtained from the gradient of the straight line (solid line), which is the best fit to the experimental data (open circles).

meV, obtained from our present optical measurements and the value reported in the literature, $91.8 \mathrm{meV}$ [32].

The scattering-limited electron mobilities $\left(\mu_{j}\right)$ were calculated using the theoretical expressions given in section 3 with the material parameters in Table 2 (Ref. $[14,34])$ and the LO phonon energy determined herein. In the numerical calculations of $\mu_{j}$ we used the value of $m^{*}=0.206 m_{0}$ for $2 \mathrm{D}$ electrons in $\mathrm{Al}_{0.25} \mathrm{Ga}_{0.75} \mathrm{~N} / \mathrm{GaN}$ heterostructures obtained from Shubnikov-de Haas effect measurements [35]. The results obtained for the temperature dependences of $\mu_{j}, \mu_{t o t}$ and $\mu_{H}$ are presented in Figure 5. According to Matthiessen's rule, the contribution of higher mobility to the total mobility (Âțtot) is less than that of lower mobility. Therefore, at low temperatures, Hall mobility of $2 \mathrm{D}$ electrons in the $\mathrm{Al}_{0.25} \mathrm{Ga}_{0.75} \mathrm{~N} / \mathrm{GaN}$ heterostructure is determined primarily by IFR scattering. We determined the lateral size $(\Lambda)$ and width $(\Delta)$ of the IFR by fitting the calculated total mobility (Equation (3)) to the Hall mobility of $2 \mathrm{D}$ electrons of $\mathrm{Al}_{0.25} \mathrm{Ga}_{0.75} \mathrm{~N} / \mathrm{GaN}$ heterostructure measured at $1.8 \mathrm{~K}$. In this procedure $\Lambda$ and $\Delta$ were taken as adjustable parameters. A good agreement between the calculated total mobility $\left(\mu_{t o t}\right)$ and the Hall mobility $\left(\mu_{H}\right)$ is obtained using $\lambda=1.5 \mathrm{~nm}$ and $\Delta=0.115 \mathrm{~nm}$ for the IFR parameters, which are comparable to those $(\Lambda=1.5$ $\mathrm{nm}$ and $\Delta=0.1 \mathrm{~nm}$ (Ref. $[10,14,34])$ ) reported previously. Figure 5 also demonstrates that the mobility of electrons in $\mathrm{Al}_{0.25} \mathrm{Ga}_{0.75} \mathrm{~N} / \mathrm{GaN}$ heterostructures is determined by IFR scattering at low temperatures and polar optical phonon scattering at high temperatures.
Table 2. Material parameters used in the calculations for the AlGaN/GaN heterostructure $[14,34]$

\begin{tabular}{ccc}
\hline & Unit & Value \\
\hline \hline Mass density & $\mathrm{kg} / \mathrm{m}^{3}$ & $6.15 \times 10^{3}$ \\
Static dielectric constant $\varepsilon_{S}$ & $\varepsilon_{0}$ & 10.4 \\
High-frequency dielectric constant $\varepsilon_{\infty}$ & $\varepsilon_{0}$ & 5.35 \\
Longitudinal acoustic phonon velocity, $u_{l}$ & $\mathrm{~m} / \mathrm{s}$ & $6.56 \times 10^{3}$ \\
Transverse acoustic phonon velocity, $u_{t}$ & $\mathrm{~m} / \mathrm{s}$ & $2.68 \times 10^{3}$ \\
Piezoelectric constant, $h_{14}$ & $\mathrm{~V} / \mathrm{m}$ & $4.28 \times 10^{9}$ \\
Deformation potential, $E_{A}$ & $\mathrm{eV}$ & 8.5 \\
Density of the 2DEG at 1.8 K & $\mathrm{m}^{-2}$ & $8.95 \times 10^{16}$ \\
Band gap energy, $E_{g}(\mathrm{GaN})$ & $\mathrm{eV}$ & 3.42 \\
Dislocation charge density $\left(N_{d i s}\right)$ & $\mathrm{cm}^{-2}$ & $1 \times 10^{10}$ \\
Impurity density, $N_{B I}$ & $\mathrm{~m}^{-3}$ & $1 \times 10^{20}$ \\
\hline
\end{tabular}

\section{Conclusion}

The energy of LO phonons in GaN was obtained from the experimental data for the temperature dependence of the Hall mobility in $\mathrm{Al}_{0.25} \mathrm{Ga}_{0.75} \mathrm{~N} / \mathrm{GaN}$ heterostructure. In addition, the Raman and IR spectra measured at room temperature were used to determine the LO phonon energy. The values obtained for the LO phonon energy from the two methods are in good agreement. The experimental data for the temperature dependence of Hall mobility were compared with calculated electron mobility to understand which scattering mechanisms limit the mobility. The results suggest that interface roughness scattering limits the electron mobility at low temperatures and at high temperatures polar optical phonon scattering is dominant.

\section{Acknowledgements}

This work is supported by the European Union under projects PHOME, ECONAM, N4E, and TUBITAK under Project Nos., 110T377, 109E301, 107A004, 107A012, and DPT under the project DPT-HAMIT, and Anadolu University under the project BAP-1001F99. One of the authors (E.O.) also acknowledges partial support from the Turkish Academy of Sciences. We would like to acknowledge Tulay TIRAS for the Raman spectroscopy measurements.

\section{References}

[1] B. A. Danilchenko et al., Phys. Status Solidi B 243, 1529 (2006)

[2] S. J. Pearton, J. C. Zolper, R. J. Shul, F. Ren, J. Appl. Phys. 86, 1 (1999) 


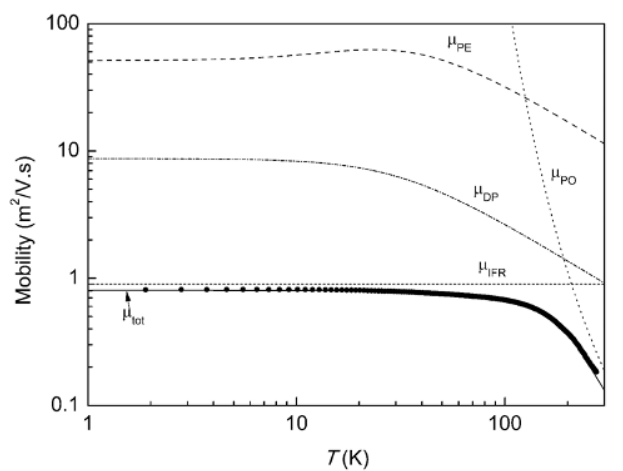

Figure 5. Temperature dependence of the Hall mobility $\left(\mu_{H}\right)$ measured at a magnetic field of $1.0 \mathrm{~T}$ (full circles) for $\mathrm{Al}_{0.25} \mathrm{Ga}_{0.75} \mathrm{~N} / \mathrm{GaN}$ heterostructure and the calculated electron mobilities: acoustic phonon deformation potential scattering mobility $\left(\mu_{D P}\right)$, piezoelectric scattering mobility $\left(\mu_{P E}\right)$, interface roughness scattering mobility $\left(\mu_{I F R}\right)$, polar optical phonon scattering mobility $\left(\mu_{P O}\right)$, total mobility $\left(\mu_{t o t}\right)$. The mobilities due to background impurity scattering and dislocation scattering mobility are not shown in here because they have very high value compared with other scattering mechanisms.

[3] S. B. Lisesivdin et al., Appl. Phys. Lett. 91 $102113(2007)$

[4] M. A. Khan, A. Bhattarai, J. N. Kuznia, D. T. Olson, Appl. Phys. Lett. 63, 1214 (1993)

[5] Y. F. Wu et al., IEEE Electr. Device L. 17, 455 (1996)

[6] A. F. M. Anwar, Shangli Wu, Richard T. Webster, IEEE T. Electron Dev. 48, 567 (2001)

[7] S. B. Lisesivdin, A. Yıldız, M. Kasap, Optoelectron. Adv. Mat. 1, 467 (2007)

[8] S. B. Lisesivdin, S. Acar, M. Kasap, S. Ozcelik, S. Gokden, E. Ozbay, Semicond. Sci. Tech. 22, 543 (2007)

[9] W. Knap et al., J. Cryst. Growth 281, 194 (2005)

[10] D. Zanato, S. Gokden, N. Balkan, B. K. Ridley W. J. Schaff, Semicond. Sci. Tech. 19, 427 (2004)

[11] W. Knap et al., J. Cryst. Growth 281, 194 (2005)

[12] S. B. Lisesivdin, S. Demirezen, M. D. Caliskan, A. Yıldız, M. Kasap, S. Ozcelik, E. Ozbay, Semicond.
Sci. Tech. 23, 095008 (2008)

[13] A. Teke et al., New J. Phys. 11, 063031 (2009)

[14] S. Gokden, Phsy. Status Solidi A, 200, 369 (2003)

[15] S. Gokden, Physica E, 23, 19 (2004)

[16] E. Tiras, S. Altinoz, M. Cankurtaran, H. Celik, N. Balkan, J. Mater. Sci. 40, 6391 (2005)

[17] H. Morkoc, Handbook of nitride semiconductors and devices, vol. 2 (WILEY-VCH Verlag GmbH \& Co. KGaA, Weinheim, 2008)

[18] B. K. Ridley, B. E. Foutz, L. F. Eastman, Phys. Rev. B 61, 16862 (1999)

[19] S. B. Lisesivdin, A. Yıldız, N. Balkan, M. Kasap, S. Ozcelik, E. Ozbay, J. Appl. Phys. 108, 013712 (2010)

[20] Y. Li, Y. Zhang, Y. Zeng, J. Appl. Phys. 109, 073703 (2011)

[21] L. Hsu, W. Walukievicz, Phys. Rev. B 56, 1520 (1997)

[22] B. L. Gelmont, M. Shur, M. Sroscio, J. Appl. Phys. 77, 657 (1995)

[23] W. Walukiewicz, H. E. Ruda, J. Lagowski, H. C. Gatos, Phys. Rev. B 30, 4571 (1984)

[24] S. Gokden, R. Baran, N. Balkan, S. Mazzucato, Physica E, 24, 249 (2004)

[25] D. Jena, A. C. Gossard, U. K. Mishra, Appl. Phys. Lett. 76, 1707 (2000)

[26] D. C. Look, H. Lu, W. J. Schaff, J. Jasinski, Z. LilientalWeber, Appl. Phys. Lett. 80, 258 (2002)

[27] K. Hirakawa, H. Sakaki, J. Yoshino, Appl. Phys. Lett. 45, 253 (1984)

[28] G. Abstreiter, M. Cardona, A. Pinczuk In: M. Cardona, G. Guntherodt (Eds.), Topics in applied physics, vol. 54 (Springer, Berlin, 1984)

[29] T. Kozawa, T. Kachi, H. Kano, Y. Taga, M. Hashimoto, J. Appl. Phys. 75, 1098 (1994)

[30] I. M. Tiginyanu et al., Phys. Rev. B 64, 233317 (2001)

[31] M. Kuball, Surf. Interface Anal. 31, 987 (2001)

[32] V. Yu. Davydov et al., Phys. Rev. B 58, 12899 (1998)

[33] E. Tiras, M. Gunes, N. Balkan, W. J. Schaff, Phys. Status Solidi B 247, 189 (2009)

[34] A. Asgari, M. Kalafi, Mater. Sci. Eng. C 26, 898 (2006)

[35] O. Celik, E. Tiras, S. Ardali, S. B. Lisesivdin, E. Ozbay, Phys. Status Solidi C 8, 1625 (2011) 